Technical note

\title{
An evaluation of the use of EBT-XD film for SRS/SBRT commissioning of a
}

\subsection{Tesla MR-Linac system}

\author{
Seng Boh Lim *, Neelam Tyagi, Ergys Subashi, Jiayi Liang, Maria Chan \\ Department of Medical Physics, Memorial Sloan Kettering Cancer Center, New York, NY 10065, United States
}

\section{A R T I C L E I N F O}

\section{Keywords:}

MR-Linac

MR-L

SBRT

QA

Film dosimetry

\begin{abstract}
A B S T R A C T
Purpose: The goal of this study was to evaluate the use of EBT-XD film for SRS/SBRT commissioning in a $1.5 \mathrm{~T}$ hybrid MR-Linac (MRL).

Method: The output factors $\left(\mathrm{S}_{\mathrm{t}}\right)$, from $1 \mathrm{x} 1,2 \mathrm{x} 2,3 \mathrm{x} 3 \mathrm{~cm}^{2}$, were measured with film in solid water. The results were compared with (1) the measurements by a PTW diamond detector (CVD) and an Exradin ${ }^{\circledR}$ A26MR ion chamber in 3D water phantom; (2) Monte Carlo calculation by Monaco TPS (MTPS) in water. The inline (IN) and crossline (CR) profiles, measured by films and the CVD, were also compared. An SRS plan with two targets was created in MTPS and was measured with EBT-XD film in a StereoPHAN ${ }^{\mathrm{TM}}$ phantom serving as an end-to-end test. The $3 \times 3$ $\mathrm{cm}^{2}$ was used for film calibration with doses ranging from 0 to $28 \mathrm{~Gy}$. Water was added to the phantom-filmphantom interface to reduce the electron-return-effect (ERE). Films were calibrated with One-scan-dosimetry protocol.

Results: The film $\mathrm{S}_{\mathrm{t}}$ were within $1.2 \%$ and $2.2 \%$ compared to other detectors and MTPS respectively. At the central B-field induced asymmetric region, films were within 0.6\% between the CVD and the MTPS, but 5-8\% differences were observed in the $40 \%-5 \%$ gradient region in CR due to ERE. The differences in localization and dose were found to be $0.6 \mathrm{~mm}$ and $3.3 \%$. The $\gamma(3 \% / 2 \mathrm{~mm}), \gamma(5 \% / 2 \mathrm{~mm}), \gamma(5 \% / 1 \mathrm{~mm})$ were $97.7 \%, 99.3 \%$, 94.6\%.

Conclusions: Films can provide accurate dosimetric results under ERE and are valuable for commissioning MRL. Using the One-scan-dosimetry protocol with EBT-XD film for MRL increases accuracy and efficiency in commissioning and QA of SRS/SBRT.
\end{abstract}

\section{Introduction}

The 1.5 Tesla (T) magnetic (B) field of Unity (Elekta, Stockholm, Sweden) MR-Linac (MRL) creates a high-intensity Lorentz force on ferrous materials rendering many conventional quality assurance (QA) detectors inoperable and poses significant challenges for clinical physicists. B-field induced Lorentz force changes the trajectories of scattering electrons in phantom resulting in additional dose deposition at the interface, asymmetric beam profiles and shifts from the central axis [1]. The effect called electron return effect or ERE is more pronounced at the tissue-air interfaces [2], and can cause significant dosimetric impact, such as higher dose in the air-water interface, in magnetic field $>0.75 \mathrm{~T}$. Even with MR-compatible equipment, the B-field, which still interacts with the scattered electrons inside the detectors, increases the measurement uncertainties during the commissioning of the treatment planning system (TPS) and adds an additional layer of complexity to the work of clinical physicists. A recent quality assurance (QA) report by the MRL Consortium provides useful guidelines for many testing methods and detector limitations [3]. In particular, the radiochromic film, which has been used extensively for conventional [4-7] and small field dosimetry $[8,9]$ in non-MRI radiation therapy, is recommended for dosimetric measurements and verification in the presence of B-field because of its nonactive property [3]. The B-field, nevertheless, still has some impacts on polymerization of the film [10-12] rendering certain conventional film techniques, such as film calibration without B-field, invalid, and non-negligible effects were found to be present in $0.35 \mathrm{~T}$ Bfield $[13,14]$. Gafchromic EBT3 film (Ashland Specialty Ingredients, Bridgewater, NJ, USA), with a recommended dose range of less than 10 $\mathrm{Gy}$, has been investigated extensively in the presence of B-field $[3,10-14]$. Film with a higher dose range, such as Gafchromic EBT-XD

\footnotetext{
* Corresponding author.

E-mail addresses: lims1@mskcc.org (S.B. Lim), tyagin@mskcc.org (N. Tyagi), subashie@mskcc.org (E. Subashi), liangj1@mskcc.org (J. Liang), chanm@mskcc.org (M. Chan).
} 
film (Ashland Specialty Ingredients, Bridgewater, NJ, USA), is more appropriate for high dose ( $>10 \mathrm{~Gy}$ ) delivery and has not been investigated yet. The goal of our study was to evaluate the use of Gafchromic EBT-XD film for the commissioning of stereotactic radiosurgery (SRS) and stereotactic body radiation therapy (SBRT) in MRL. To the best of our knowledge, this is the first investigation of small field dosimetry with Gafchromic EBT-XD film on a 1.5T MRL.

This paper focuses on evaluating the feasibility of using EBT-XD film in the commissioning of small fields in MRL in order to improve efficiency and ensure the safe delivery of SRS/SBRT in MRL. Following the introduction, film calibration under B-field is described, and the comparison of the film along with MR-compatible detectors and Monte-Carlo based dose calculations in phantom is presented. Finally, the dosimetry and localization accuracy of an end-to-end test (E2E) with film are discussed.

\section{Materials and methods}

\subsection{System description}

The Unity MRL is a combined 1.5T MR scanner and 7X-FFF Linac. The source-to-axis distance (SAD) is $143.5 \mathrm{~cm}$ (see Fig. 1). The TPS used in this study was Monaco TPS (MTPS) v.5.40.01 (Elekta, Stockholm, Sweden) which is a Monte Carlo-based dose calculation system. All dose calculations were performed using an isotropic dose grid of $1.0 \mathrm{~mm}$ and a statistical uncertainty of $0.5 \%$.

\subsection{Study design}

An E2E test, an evaluation process of a complete clinical workflow, including CT scanning, treatment planning, pre-treatment imaging, online adaptation, and delivery, was performed with films and a StereoPHAN $^{\mathrm{TM}}$ (Sun Nuclear Corp, Melbourne, FL, USA). Films were placed inside an MR-compatible film insert. The exposed films were scanned and calibrated using the One-scan protocol and film dosimetry for SRS/ SBRT $[4,5,7,9]$ to improve calibration accuracy by minimizing the scanto-scan variation and using a small field for calibration. The output factors $\left(\mathrm{S}_{\mathrm{t}}\right)$, measured from the calibrated films, were compared with those measured with microDiamond (CVD) (PTW, Freiburg, Germany) and Exradin ${ }^{\circledR}$ A26MR ion chamber (Standard Imaging, Middleton, WI, USA). The profiles measured with CVD and Semiflex 3D (PTW, Freiburg, Germany) were also compared to those measured with film. The recorded doses on the calibrated E2E films were registered and compared with the Monaco plan using a gamma $(\gamma)$ index [15] of $3 \% / 2 \mathrm{~mm}, 5 \% /$ $2 \mathrm{~mm}$, and $5 \% / 1 \mathrm{~mm}$.

\subsection{Dosimetric measurements tools}

The dosimetry tools used in this study include Gafchromic EBT-XD film (lot \# 04282002) (Ashland Specialty Ingredients, Bridgewater, NJ, USA), CVD, and A26MR micropoint ion chamber. Solid Water ${ }^{\circledR}$ for the film calibration and irradiation; a BeamScan ${ }^{\circledR}$ MR $3 \mathrm{D}$ water tank (PTW, Freiburg, Germany) was also used for the CVD and A26MR measurements. The E2E test was conducted using a StereoPHAN system.

\subsection{Film calibration with $B$-field on}

\subsubsection{Feasibility}

First, to test the validity of Gafchromic EBT-XD film in measuring small fields in the B-field, a set of EBT-XD film strips $\left(10 \times 13 \mathrm{~cm}^{2}\right)$ were exposed on the Unity MRL at open field sizes of $1 \times 1,2 \times 2,3 \times 3$, and 10x10 $\mathrm{cm}^{2}$ with a fixed monitor unit (MU) of 1774 using $7 \mathrm{MV}$ flattening-filterfree (FFF) beam. $1774 \mathrm{MU}$, which is $16.1 \mathrm{~Gy}$ for $3 \times 3 \mathrm{~cm}^{2}$, was chosen to represent the mid-range of the calibration curve (see 2.4.2) and to produce sufficient optical density for the smallest field $\left(1 \times 1 \mathrm{~cm}^{2}\right)$ exposure with EBT-XD film. For each delivery, a film was placed inside a Solid Water ${ }^{\circledR}$ phantom stack (Sun Nuclear Corp, Melbourne, FL, USA) at a depth of $5 \mathrm{~cm}$ with $10 \mathrm{~cm}$ back-up phantom at a source-axis distance (SAD) of $143.5 \mathrm{~cm}$.

\subsubsection{Calibration}

A triple-channel film calibration up to $28 \mathrm{~Gy}$ was generated based on a machine-specific reference of $0.908 \mathrm{cGy} / \mathrm{MU}$ with 7XFFF for Elekta Unity, at $143.5 \mathrm{~cm} \mathrm{SAD,} \mathrm{d}=5 \mathrm{~cm}, 3 \times 3 \mathrm{~cm}^{2} \mathrm{MLC} / \mathrm{jaw}$ setting instead of open $10 \times 10 \mathrm{~cm}^{2}$ field. The $3 \times 3 \mathrm{~cm}^{2}$ field size was used for film calibration with doses ranging from 0 to $28 \mathrm{~Gy}$ to minimize the impact of field size $[4,9]$. Using the built-in dose sequence calculator in FilmQA ${ }^{\mathrm{TM}}$ Pro (Ashland Specialty Ingredients, Bridgewater, NJ, USA), a predetermined maximum dose of $28 \mathrm{~Gy}$, the subsequence calibration doses were given as $21.38,16.11,11.78,8.14,5.04,2.35$, and $0 \mathrm{~Gy}$, respectively covering the typical dose range of an SRS/SBRT delivery. The corresponding MU were 3084, 2355, 1774, 1297, 896, 555, 259, and 0, respectively. The calibration curve was saved as a master calibration curve for the same film lot with the One-scan protocol [5] in this study.

To minimize the magnetic field effect on the film polymerization, all the calibration films were exposed on the MRL [10-12]. All films were scanned with a resolution of 150 dpi with Epson 10000XL (Epson, Los Alamitos, CA, USA) using the One-scan method [5] with FilmQA Pro (see screenshot in Fig. 2). Two additional reference films, with known doses (20 Gy and $0 \mathrm{~Gy}$ ), were scanned, together with each of the small open fields $\left(3 \times 3 \mathrm{~cm}^{2}\right)$ and the clinical SRS films to eliminate the scanner and temperature effects $[4,5,7,9]$. The reference films were used to

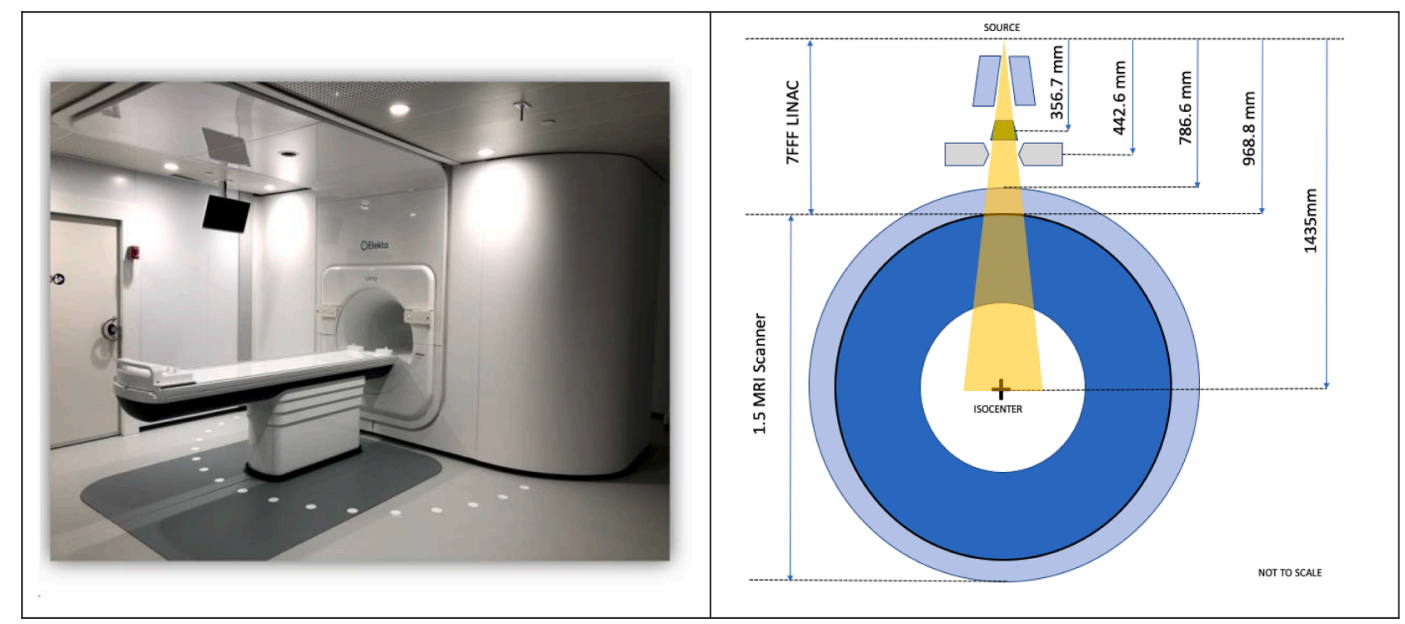

Fig. 1. Left: Picture of the MRL room; Right: Structure of the MRL. 


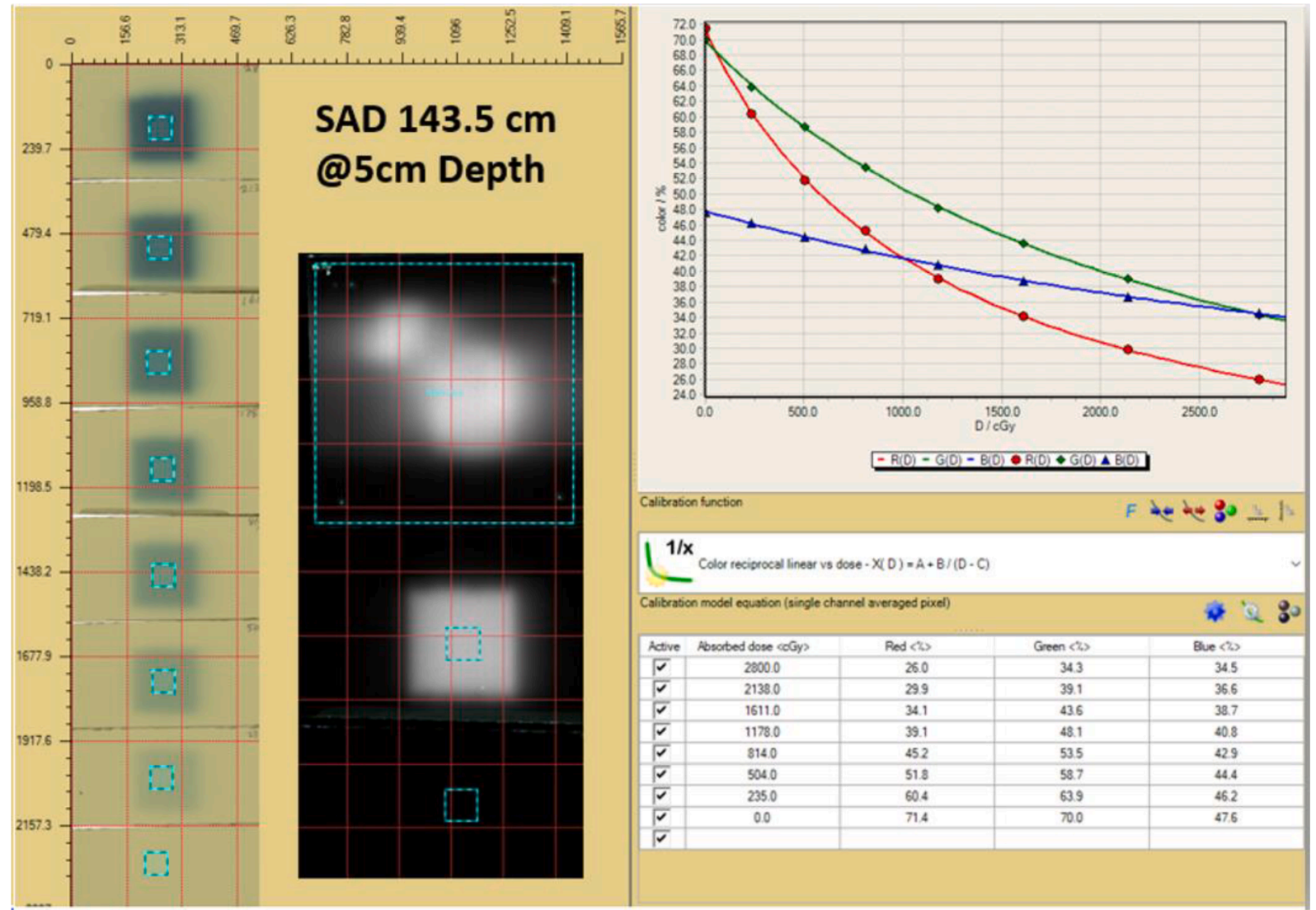

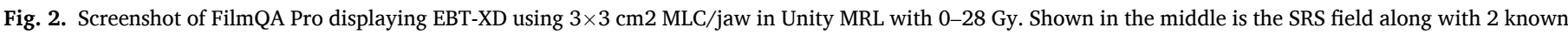
doses (20 Gy and 0 Gy).

rescale the calibration function for the same scan conditions. Note that the One-scan protocol [5] was not developed for small fields but the methodology can be applied to small field dosimetry except for the different requirements on calibration field size $[4,9]$.

\subsection{Output factors and profiles of small open fields}

For the small field verification, output factors $\left(\mathrm{S}_{t}\right)$ and profiles for penumbra assessment were measured at the depth of $5 \mathrm{~cm}$ for field sizes of $1 \times 1,2 \times 2,3 \times 3 \mathrm{~cm}^{2}$. The average dose of an area of $5 \times 5 \mathrm{~mm}^{2}$ around the central axis of each field size was measured. The dose ratios, relative to $3 \times 3 \mathrm{~cm}^{2}$, were first calculated and daisy-chain to MTPS calculated $S_{t}$ of $3 \times 3 \mathrm{~cm}^{2}$ to determine the film-based $S_{t}$. The MTPS calculated $S_{t}$ for $3 \times 3$ $\mathrm{cm}^{2}$ used is 0.914. During the placement of each film in the Solid Water stack, a few drops of water were added to the film (see Fig. 3(a)) to minimize the air gap between the film and the phantom slabs to reduce the potential uncertainties from the ERE. Films were also submerged in water for the same measurement setup to evaluate the efficacy of the water droplet technique (see Fig. 3(b)). Measurements were repeated using CVD and A26MR in the 3D water tank (see Fig. 3(c)). Recommended small field detector output corrections [8] were applied to the readouts.

\subsection{E2E test of SRS/SBRT}

SRS/SBRT multi-met lesions (2 targets ranged 10-20 mm) were planned with a single isocenter using MTPS based on the planning CT imaging of the StereoPHAN. Fig. 4 (Left) shows an image of the StereoPHAN phantom. A piece of film was placed in the phantom cube and an adaptive plan was delivered to the phantom and measured (Fig. 4 Right).
In addition to the 2-target E2E case, three more SRS/SBRT cases were planned and delivered to examine the reproducibility. Table 1 summarizes all 4 cases. Fig. 5 shows the sagittal view of the CT of the StereoPHAN with the planning targets in Case 1 . All prescriptions were normalized to $100 \%$ isodose line.

\section{Results and discussion}

\subsection{Efficacy of water droplet technique in B-field}

Fig. $6 a$ and $b$ show the effect of profiles measured with film under water droplets and no water condition. Relative to MTPS, dosimetric uncertainties of order $2.2 \%$ and $3.8 \%$ were observed in film measurements in the radial and transverse directions. With water, however, the measured profiles agreement to MTPS improved to within $0.7 \%$ and $1.3 \%$ in the radial and transverse directions. No significant differences, on average within $0.3 \%$, were found between the profile measured on film when the film was placed in solid water using the water droplet technique and when the solid water stack along with the film was placed in water. Fig. $6 \mathrm{c}$ and d show an example of the profile comparison measured with the two techniques. All the profiles were registered to the TPS profile and normalized to the center of the beam. Within the in-field region, films measured in both techniques show similar noise levels. In the penumbra region, differences were not significant and were within film measurement uncertainty. The results indicate the water droplet technique was able to eliminate the air gap around the film in the phantom without the need for submerging the solid water stack in a water tank. 

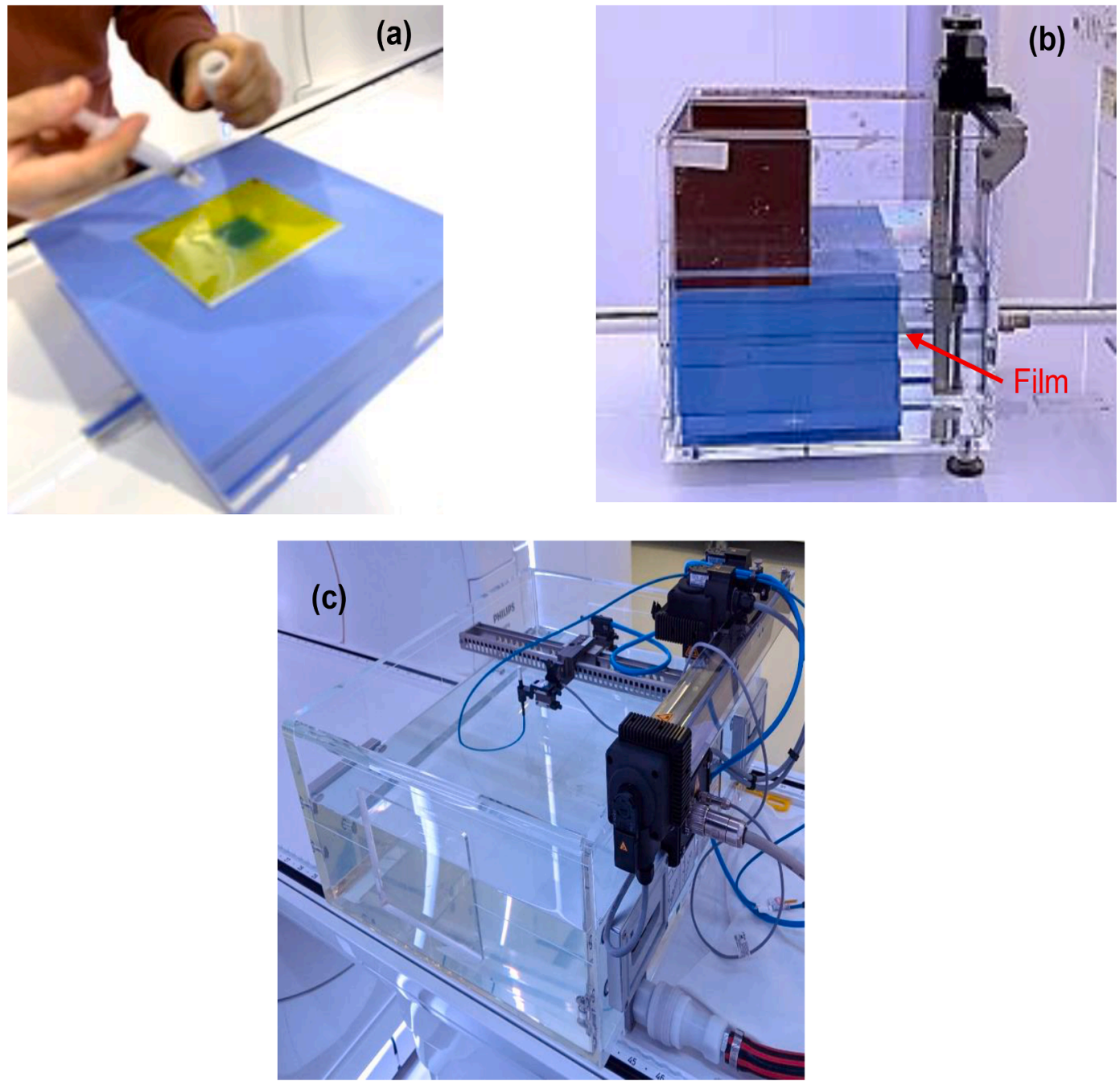

Fig. 3. (a) Adding drops of water to the film before adding the phantom slabs on the top; (b) \&(c) PTW BeamScan MR 3D water tank set up on the MRL couch.

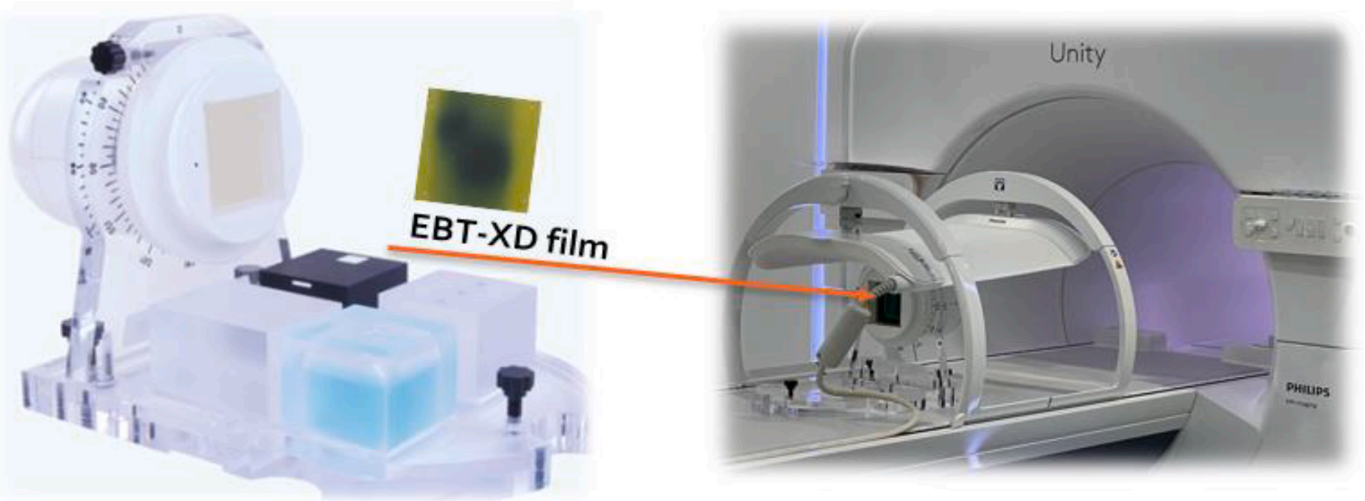

Fig. 4. Left: StereoPHAN with film in the water cubes; Right: The StereoPHAN in the MR coil to be imaged to register with the treatment plan before SRS delivery.

\subsection{Output factors and profiles of small open fields}

The measured $\mathrm{S}_{\mathrm{t}}$, measured by films, were found to be 0.676 and 0.848 for $1 \mathrm{x} 1$ and $2 \mathrm{x} 2 \mathrm{~cm}^{2}$. Comparing to CVD and A12MR, the $S_{t}$ were within $1.2 \%$ and $1.1 \%$ for $1 \mathrm{x} 1$ and $2 \times 2 \mathrm{~cm}^{2}$ respectively. The corresponding $S_{t}$ for $1 \times 1$ and $2 \times 2 \mathrm{~cm}^{2}$ from MTPS were found to be 0.693 and 0.862 which were within $1.6 \%$ and $2.3 \%$ of the $S_{t}$ measured with films (see Fig. 7 Left). The $S_{t}$ by CVD and A12MR were found to be within
$1.3 \%$ and $3.4 \%$ from MTPS within the same field sizes. Within the central B-field induced asymmetric region, films were within $0.6 \%$ on average from the CVD and the MTPS. Radial profiles, for field sizes $1 \times 1$ to $3 \times 3 \mathrm{~cm}^{2}$, agree with CVD and MTPS to within $<1 \mathrm{~mm}$. The center of the beam was found to be shifted to the right by $2-3 \mathrm{~mm}$ in the transverse direction (see Fig. 7 Right and Fig. 8). Larger differences, in the order of $5-8 \%$, were also observed between films and CVD in the $40 \%$ $5 \%$ gradient region in crossline profiles (see Fig. 8 Right). 
Table 1

A summary of the clinical cases used in this study.

\begin{tabular}{llll}
\hline Case & Type & Target Size $\left(\mathrm{cm}^{3}\right)$ & Target Prescription Dose (Gy) \\
\hline 1 & Two Targets SRS & $\begin{array}{l}\text { Target } 1: 1.6 \\
\text { Target 2: } 7.4\end{array}$ & $18 \mathrm{~Gy}$ \\
& & 21.8 & $15 \mathrm{~Gy}$ \\
2 & Single Target SRS & 100.9 & $15 \mathrm{~Gy}$ \\
3 & SBRT & 101.1 & $15 \mathrm{~Gy}$ \\
4 & SBRT & & \\
\hline
\end{tabular}

Similar dose differences in the penumbra region were observed in the transverse profiles in the $1 \times 1$ and $2 \times 2 \mathrm{~cm}^{2}$ apertures. These discrepancies were only observed in the direction perpendicular to the B-field (transverse) where the ERE has the strongest influence.

Investigating the ERE impacts under the full lateral electron equilibrium condition, three detectors, an ion chamber, the CVD, and the film, were irradiated with a larger $10 \times 10 \mathrm{~cm}^{2}$ field and compared (Fig. 9). In the inline direction, the CVD and film showed sharper penumbra similar to a previous study [1]. In the transverse direction, where a significant ERE is present, film showed a different profile from the CVD in the lower dose portion (40-5\%) of the penumbra consistent with the small field measurements.

Scattered electrons, under the influence of B-field, were bent in a circular trajectory resulting in hitting the detector (both CVD and film) at angles that could be significantly different from the incident primary beam. CVD, under the influence of high B-field, has been shown to have significant angular dependence in the order of $10 \%$ at $60^{\circ}[1]$. In the penumbra region, where the scattered electrons can be the dominant component of the beam, the CVD can show a different behavior from the films where no angular dependence was found [16]. As the MTPS was modeled using the CVD data, the angular dependence of CVD likely affected the calculation beam modeling. In addition, we have eliminated the film scanning orientation as a contributing factor for the discrepancy in the low dose region of the crossline profiles by comparing the $3 \times 3 \mathrm{~cm}^{2}$ profiles with the film scanned in both portrait and landscape directions. Thus, the profiles extracted from films could be closer to reality under the B-field influence. Therefore, films should be considered for the MTPS commissioning or at the very least a verification tool.

\section{E2E test of SRS/SBRT}

Fig. 10 shows an example of an overlay of the film and the Monaco treatment planning system calculation and the corresponding dose differences analysis. The differences in localization and dose were found to be $0.6 \mathrm{~mm}$ and $3.3 \%$. The $\gamma(3 \% / 2 \mathrm{~mm}), \gamma(5 \% / 2 \mathrm{~mm})$, and $\gamma(5 \% / 1 \mathrm{~mm})$ within $50 \%$ target dose region were $97.7 \%, 99.3 \%$, and $94.6 \%$. Within $10 \%$ target dose region, the $\gamma(3 \% / 2 \mathrm{~mm}), \gamma(5 \% / 2 \mathrm{~mm})$, and $\gamma(5 \% /$ $1 \mathrm{~mm}$ ) were $95.6 \%, 100.0 \%$, and $96.3 \%$.

Similar to the static apertures, differences in the penumbra regions were observed, and the MTPS under-estimated in the lower dose portion of the penumbra which can also be better visualized in Fig. 11. Although the results satisfy the dosimetric and localization criteria of $5 \%$ and 1 $\mathrm{mm}$ respectively from the AAPM-RSS MPPG 9a guideline [17], differences in the penumbra region should be carefully assessed for potential clinical impacts.

The range of $\gamma$ analysis of all the cases with $3 \% / 2 \mathrm{~mm}, 5 \% / 2 \mathrm{~mm}$, and $5 \% / 1 \mathrm{~mm}$ with a threshold of $10 \%$ were found to be [94.9, 100.0], [99.5, $100.0]$, and $[96.3,99.9]$ respectively (Table 2). The lower $\gamma(5 \% / 1 \mathrm{~mm})$ results of case 1 were attributed to the failure at the penumbra regions.

During the MRL SRS/SBRT commissioning, we found the One-scan protocol [5], a well-established technique for Gafchromic films in conventional radiotherapy, gave high spatial resolution and efficiency gains by calibrating and irradiating under the same magnetic field influence. In the 1.5T Unity MRL system, crossline beam profiles have been reported to be asymmetric and shifted from the central axis because of the strong Lorentz force [1]. Both profile measurements, film in phantom and CVD in water, were within $2 \%$ agreement and showed asymmetrically and shifted crossline beam profiles. Deviations between film and CVD occurred on the right side of the crossline profiles at the $40 \%-5 \%$ gradient region. As penumbra contribution is significantly higher in smaller fields, the dosimetric uncertainty likely increases with decreasing field size. The film-based output factor measurements were
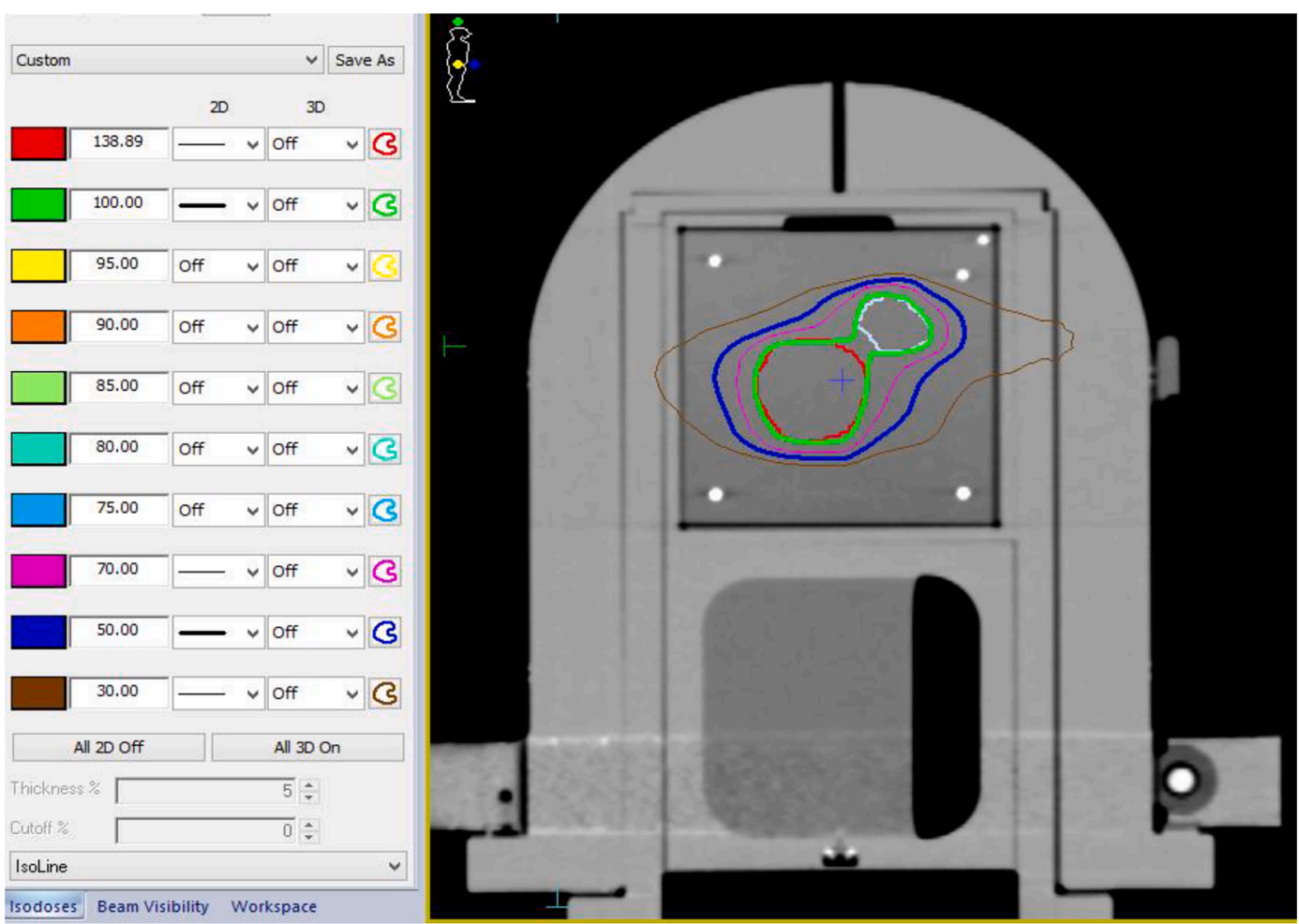

Fig. 5. The sagittal view of the StereoPHAN CT scan with the planning targets in Case 1 . 

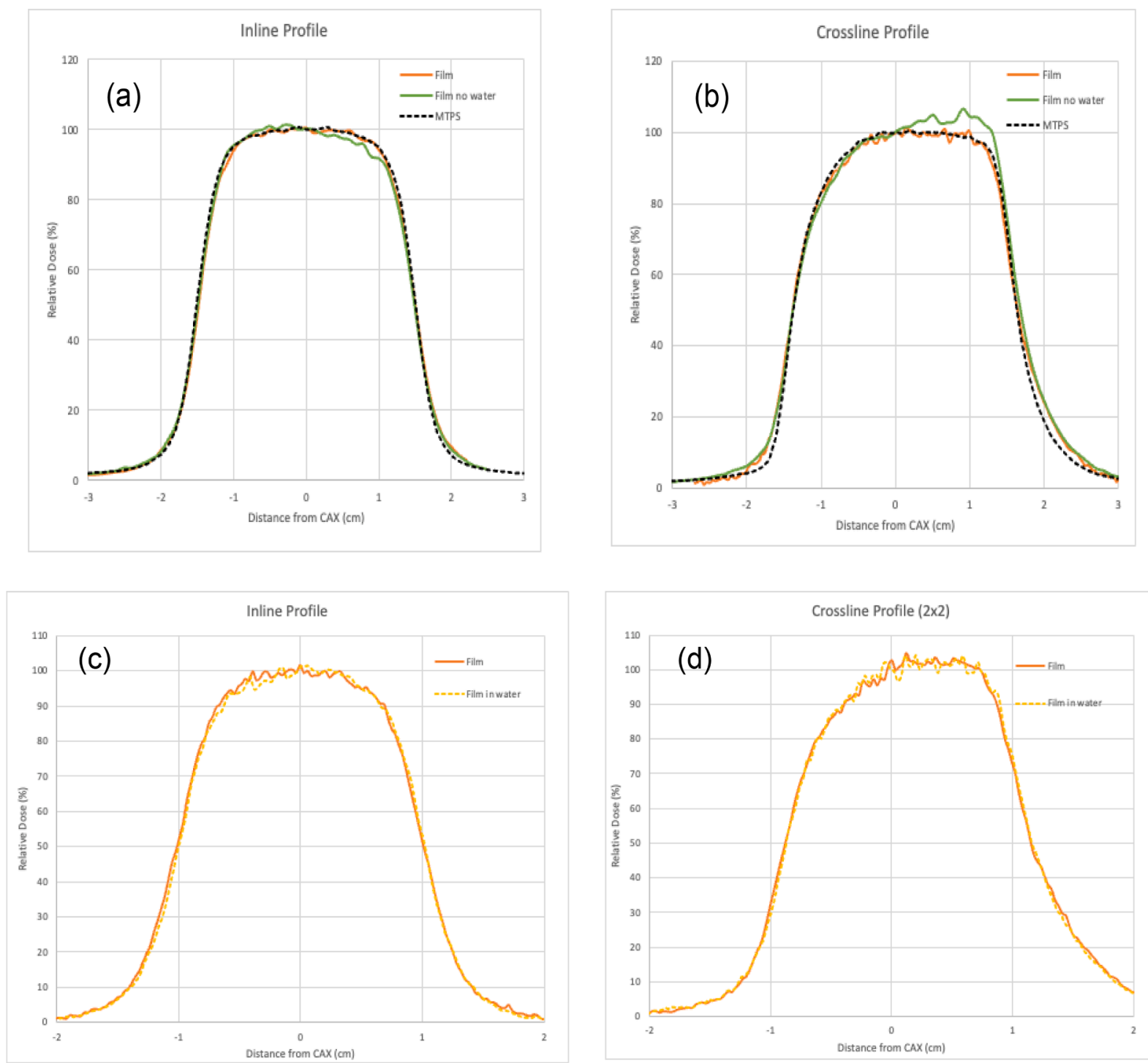

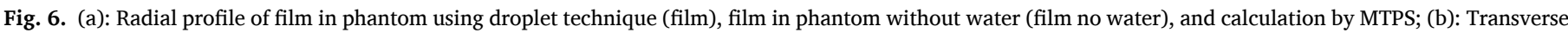

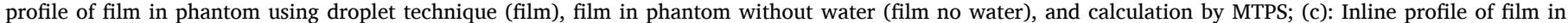

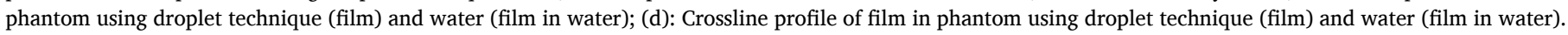

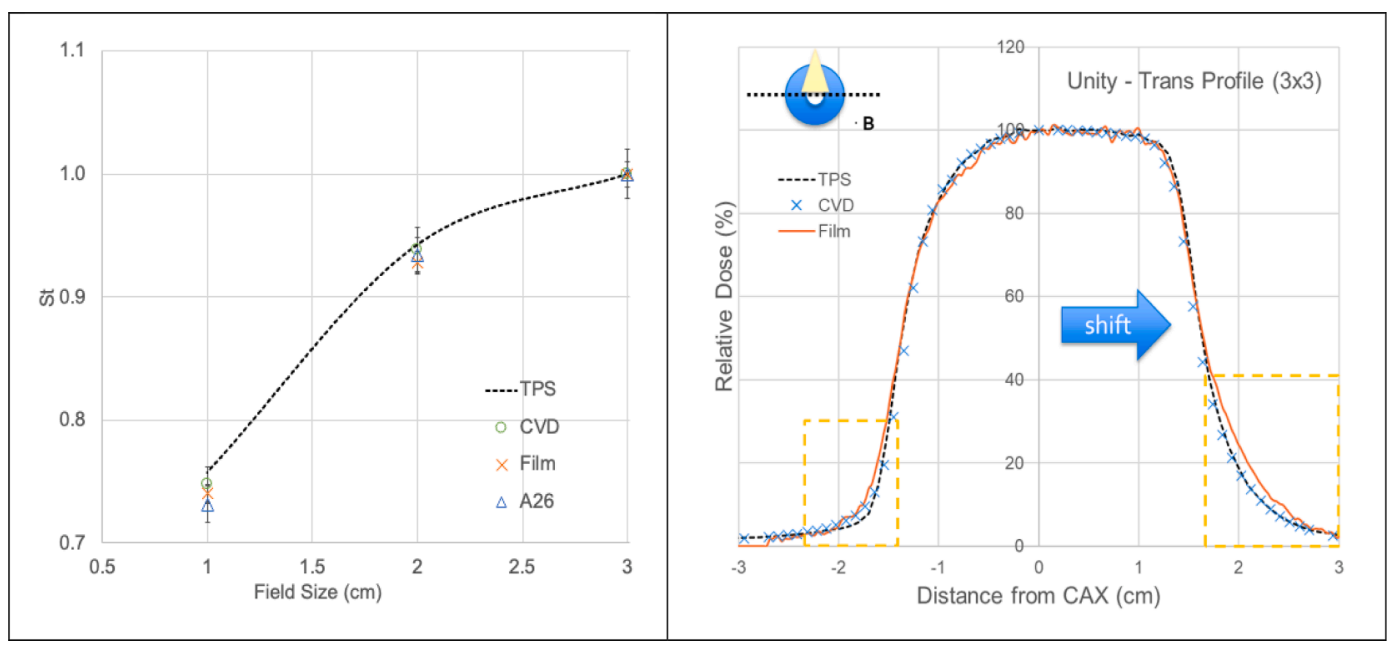

Fig. 7. Left: St field size output factors (TPS, CVD, Film); Right: Crossline profiles comparison (TPS, CVD, Film). 

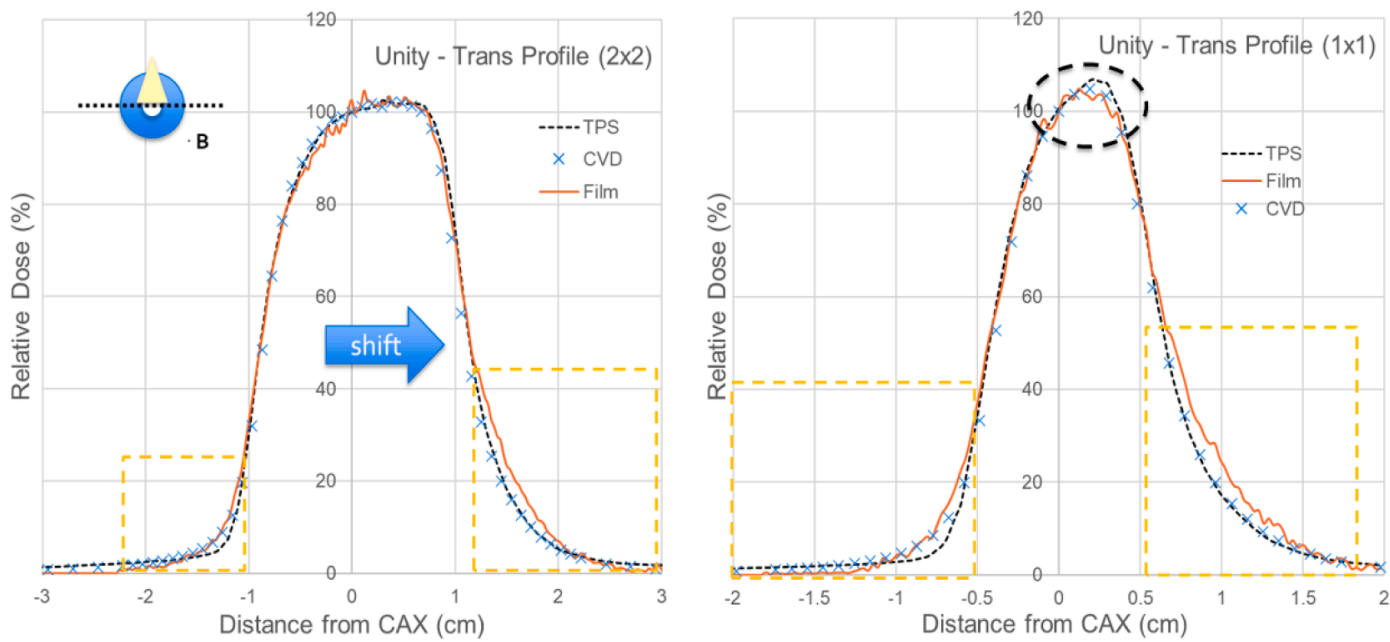

Fig. 8. Left: $2 \times 2 \mathrm{~cm} 2$ Crossline profiles comparison (TPS, CVD, Film); Right: $1 \mathrm{x} 1 \mathrm{~cm} 2$ Crossline profiles comparison (TPS, CVD, Film).

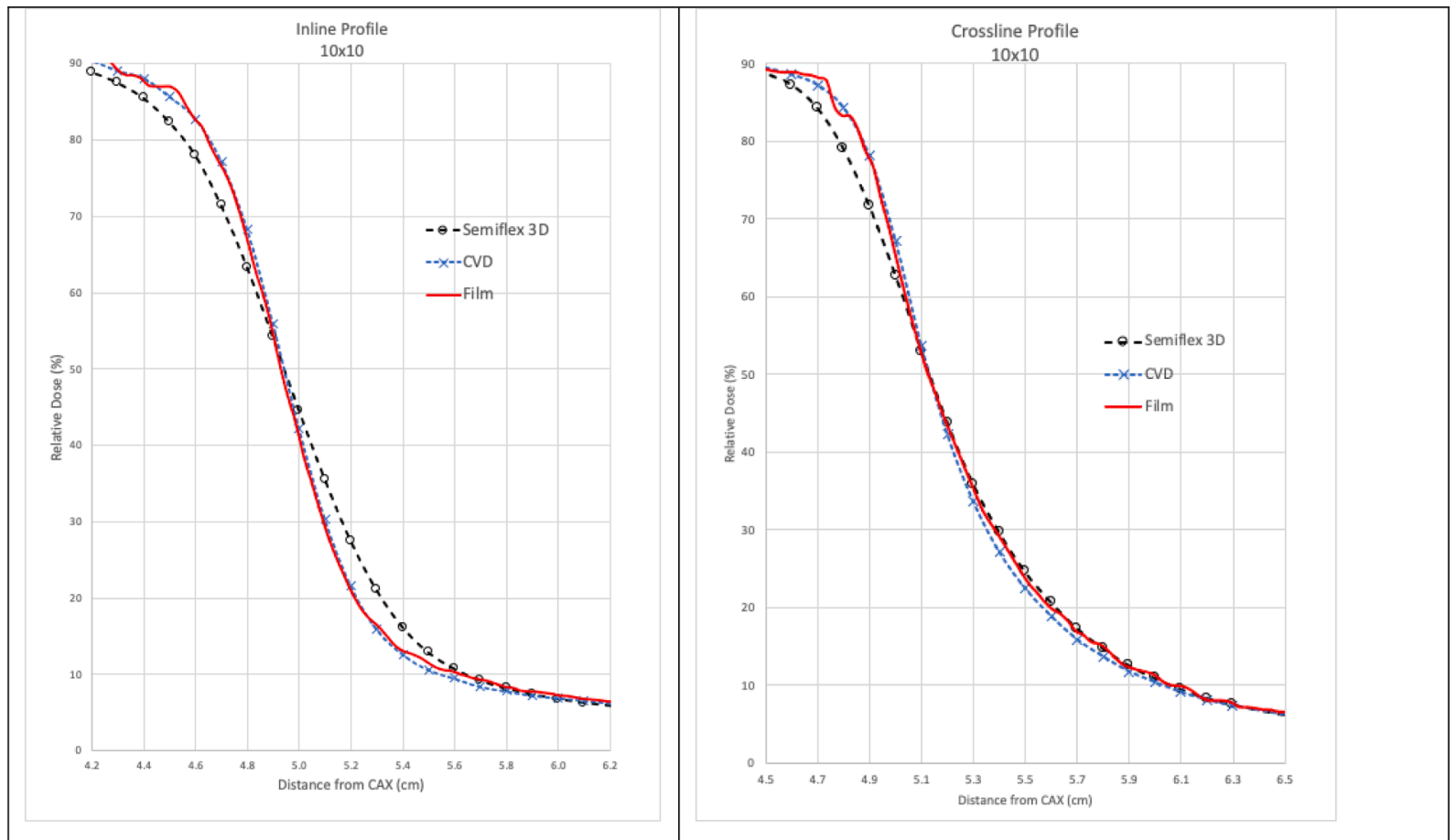

Fig. 9. Penumbrae of a 10x10 field measured with Semiflex 3D, CVD, and film; Left: Inline profiles comparison; Right: Crossline profiles comparison.

also validated with two independent small detectors recommended by IAEA TRS-483 [8]. A clinical multiple-Mets SRS plan with a prescribed dose of $18 \mathrm{~Gy}$ to targets was delivered to the StereoPHAN phantom with a film insert. In this study, we did not apply the lateral response artifact (LRA) correction [18] to the SRS films' scanning due to the small field sizes used in this study and the less LRA of EBT-XD film at higher doses (i.e., > 10 Gy) [19]. Further investigation of using other small field detectors, such as plastic scintillator, and an independent Monte Carlo verification [20] will be beneficial as only a limited number of MR compatible detectors are currently available for clinical use.

\section{Conclusions}

Our investigation showed that the output factors and in-field profiles agree within $2.2 \%$ in the high dose region among film, and PTW diamond detector (CVD) as well as micropoint ion chamber (A26MR) measured in water. However, the ERE was found to be a contributing factor (in both CVD and film or just CVD) to the uncertainty in the penumbra region perpendicular to the B-field for both the square and SRS fields. With appropriate care, films can provide accurate dosimetric results under ERE and is a valuable commissioning tool for MRL. The use of the One-scan dosimetry protocol with EBT-XD film for MRL increases accuracy and efficiency in commissioning and QA of SRS/SBRT and small field dosimetry.

Funding: This work was partly supported by the NIH/NCI Cancer Center Support Grant P30 CA008748.

\section{Declaration of Competing Interest}

The authors declare the following financial interests/personal relationships which may be considered as potential competing interests: The department has a master research agreement with Elekta, the 


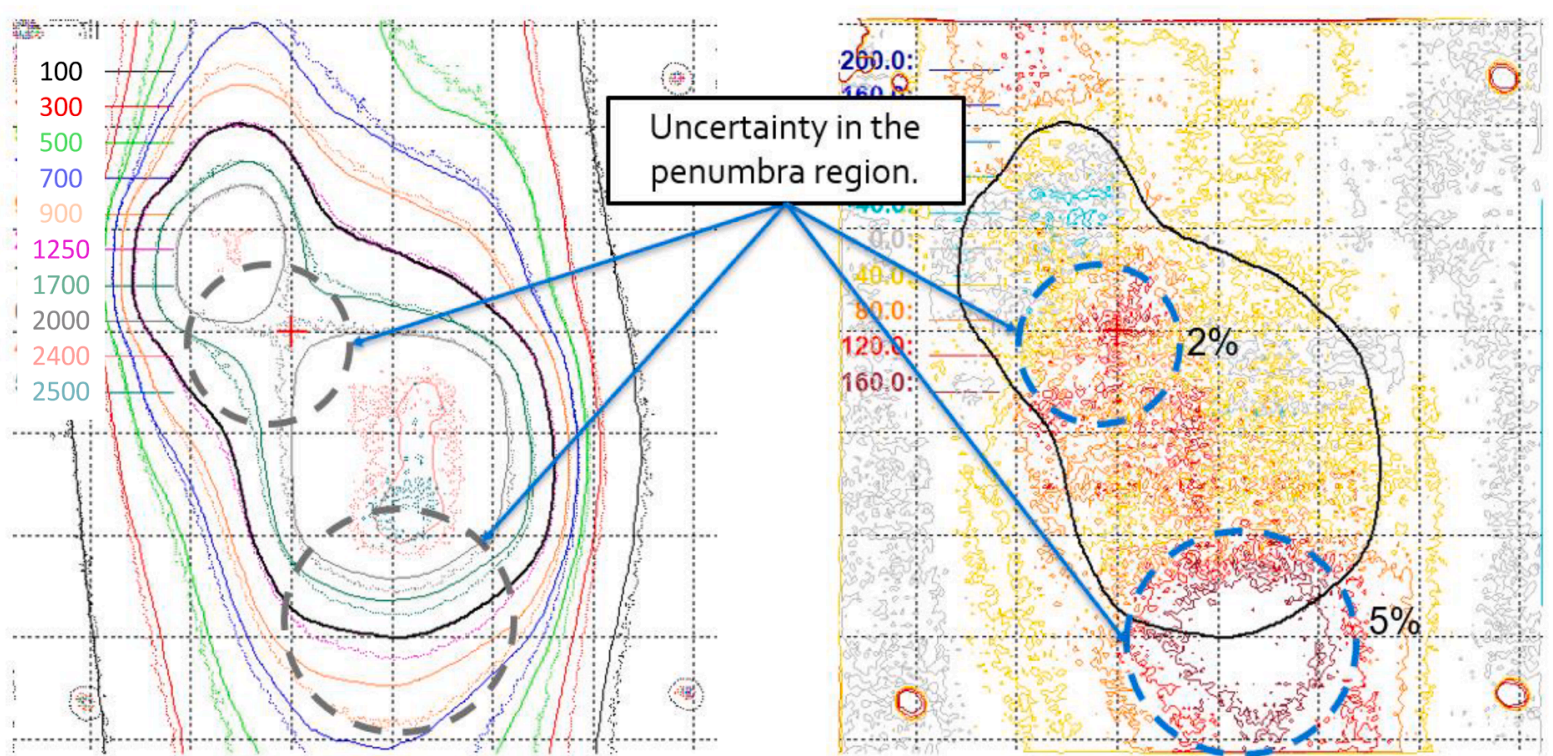

Fig. 10. Left: TPS and Film Overlay (ROI - black 50\% isodose line); Right: Dose difference between film and TPS.

Profile Analysis normalized to average values $(-0.8$ to $1.2 \mathrm{~cm})$

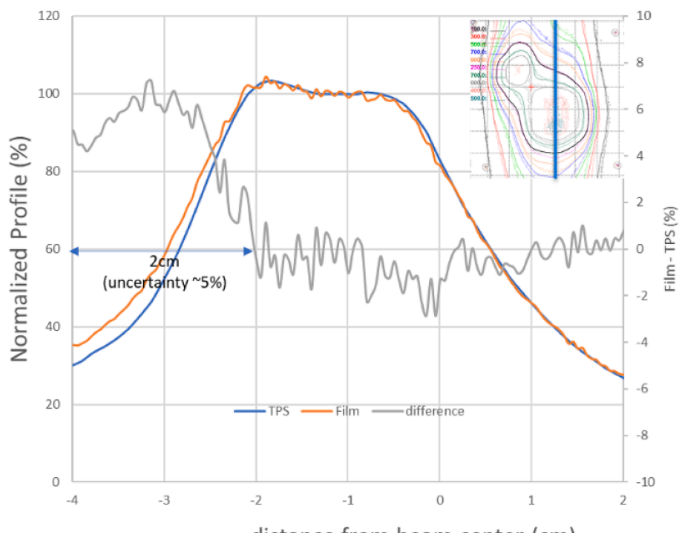

distance from beam center $(\mathrm{cm})$
Diagonal Profile Analysis

normalized to average values $(-1.95$ to $-2.05 \mathrm{~cm})$

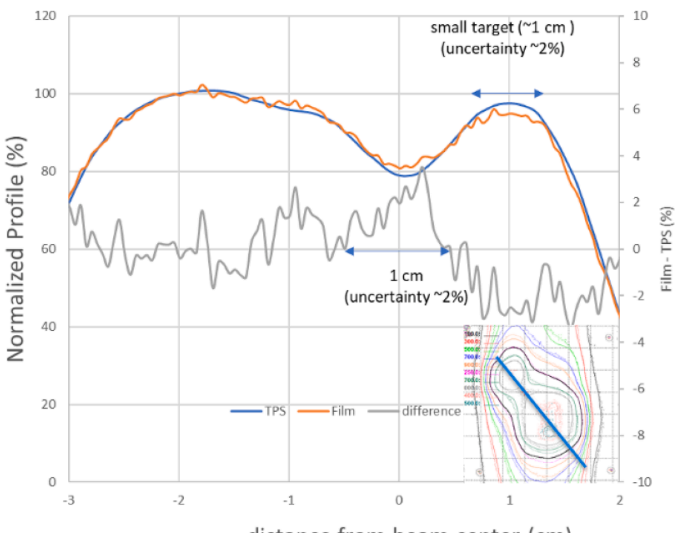

distance from beam center $(\mathrm{cm}$

Fig. 11. Left: TPS and Film Overlay (ROI - black 50\% isodose line); Right: Dose difference between film and TPS.

Table 2

The table summarizes the results of the $\gamma$ analysis of four cases of SRS/SBRT delivery.

\begin{tabular}{lccl}
\hline \multirow{2}{*}{ Case } & \multicolumn{2}{l}{$\gamma($ threshold $=10 \%)$} & \\
\cline { 2 - 4 } & $3 \% / 2 \mathrm{~mm}$ & $5 \% / 2 \mathrm{~mm}$ & $5 \% / 1 \mathrm{~mm}$ \\
\hline $1^{*}$ & 95.6 & 100.0 & 96.3 \\
2 & 100.0 & 99.9 & 99.9 \\
3 & 96.0 & 99.5 & 99.4 \\
4 & 94.9 & 100.0 & 99.0 \\
\hline
\end{tabular}

"Case 1 is a single-iso SRS plan with 2 targets.

manufacturer of Unity MR-Linac; Maria Chan has a research grant from Ashland, Inc., the manufacturer of Gafchromic film.

\section{References}

[1] Woodings SJ, Wolthaus JWH, van Asselen B, de Vries JHW, Kok JGM, Lagendijk JJW, et al. Performance of a PTW 60019 microDiamond detector in a 1.5 T MRI-linac. Phys Med Biol. 2018;63(5):63, 05nt4.
[2] Raaijmakers AJE, Raaymakers BW, Lagendijk JJW. Magnetic-field-induced dose effects in MR-guided radiotherapy systems: dependence on the magnetic field strength. Phys Med Biol. 2008;53(4):909-23.

[3] Roberts DA, Sandin C, Vesanen PT, Lee H, Hanson IM, Nill S, et al. Machine QA for the Elekta Unity system: A Report from the Elekta MR-linac consortium. Med Phys. 2021;48(5). https://doi.org/10.1002/mp.v48.510.1002/mp.14764.

[4] M.F. Chan. Recent advancements and applications in dosimetry. 2018.

[5] Lewis D, Micke A, Yu X, Chan MF. An efficient protocol for radiochromic film dosimetry combining calibration and measurement in a single scan. Med Phys. 2012;39(10):6339-50.

[6] Micke A, Lewis DF, Yu X. Multichannel film dosimetry with nonuniformity correction. Med Phys. 2011:38(5):2523-34.

[7] Niroomand-Rad A, Chiu-Tsao S-T, Grams MP, Lewis DF, Soares CG, Van Battum LJ, et al. Report of AAPM Task Group 235 Radiochromic Film Dosimetry: An Update to TG-55. Med Phys. 2020;47(12):5986-6025.

[8] Dosimetry of Small Static Fields Used in External Beam Radiotherapy. Vienna: INTERNATIONAL ATOMIC ENERGY AGENCY. 2017

[9] Chan M, Song Y. Film dosimetry for linear accelerator-based stereotactic radiosurgery and stereotactic body radiation therapy. Role and Applications in Radiation Dosimetry. 2017:165-84.

[10] Billas I, Bouchard H, Oelfke U, Duane S. The effect of magnetic field strength on the response of Gafchromic EBT-3 film. Phys Med Biol. 2019;64(6):64. 06nt3.

[11] Reynoso FJ, Curcuru A, Green O, Mutic S, Das IJ, Santanam L. Technical Note: Magnetic field effects on Gafchromic-film response in MR-IGRT. Med Phys. 2016; 43(12):6552-6. 
[12] Volotskova O, Fang X, Keidar M, Chandarana H, Das IJ. Microstructure changes in radiochromic films due to magnetic field and radiation. Med Phys. 2019;46(1): 293-301.

[13] Cusumano D, Teodoli S, Greco F, Fidanzio A, Boldrini L, Massaccesi M, et al Experimental evaluation of the impact of low tesla transverse magnetic field on dose distribution in presence of tissue interfaces. Phys Med. 2018;53:80-5.

[14] Gungor G, Korkmaz L, Kayalilar Sr N, Aydin G, Yapici B, Zoto Mustafayev T, et al Multichannel Film Dosimetry for Quality Assurance of Intensity Modulated Radiotherapy Treatment Plans Under 0.35 T Magnetic Field. Cureus. 2020:12. e7334.

[15] Low DA, Dempsey JF. Evaluation of the gamma dose distribution comparison method. Med Phys. 2003;30(9):2455-64.
[16] van Battum LJ, Hoffmans D, Piersma H, Heukelom S. Accurate dosimetry with GafChromic EBT film of a 6 MV photon beam in water: what level is achievable? Med Phys. 2008;35(2):704-16.

[17] Halvorsen PH, Cirino E, Das IJ, Garrett JA, Yang J, Yin FF, et al. AAPM-RSS Medical Physics Practice Guideline 9.a. for SRS-SBRT. J Appl Clin Med Phys. 2017; 18:10-21.

[18] Lewis D, Chan MF. Correcting lateral response artifacts from flatbed scanners for radiochromic film dosimetry. Med Phys 2015;42(1):416-29.

[19] Lewis DF, Chan MF. Technical Note: On GAFChromic EBT-XD film and the lateral response artifact. Med Phys 2016;43(2):643-9.

[20] Alhamada H, Simon S, Gulyban A, Gastelblum P, Pauly N, VanGestel D, et al. Monte Carlo as quality control tool of stereotactic body radiation therapy treatment plans. Phys Med. 2021;84:205-13. 